\title{
Left atrial appendage function in patients with atrial flutter
}

Heyder Omran, Werner Jung, Rami Rabahieh, Dean MacCarter, Stefan Illien, Barbara Rang, Andreas Hagendorff, Rainer Schimpf, Berndt Lüderitz

\begin{abstract}
Objective-To determine whether echocardiographic markers of thromboembolic risk differ between patients with pure atrial flutter and patients with atrial flutter and intermittent atrial fibrillation. Design-Patients with atrial flutter were followed up prospectively for 12 months to identify intermittent atrial fibrillation. After the follow up period, transthoracic and multiplane transoesophageal echocardiography were performed to assess left atrial chamber and appendage size, peak emptying velocities, and emptying fraction of the left atrial appendage. The presence of spontaneous echo contrast was also determined.
\end{abstract}

Setting-Tertiary cardiac care centre.

Patients-20 consecutive patients with atrial flutter; 11 healthy subjects in sinus rhythm served as controls.

Results-Intermittent atrial fibrillation was documented in 11 patients by Holter monitoring or surface ECG; atrial fibrillation was not found in the other nine patients. Compared with the patients with pure atrial flutter, patients with atrial flutter and intermittent atrial fibrillation had larger left atrial chamber (mean (SD) $4.5(0.6)$ v $3.8(0.5) \mathrm{cm} ; 95 \%$ confidence interval 0.2 to $1.2 ; \quad P=0.01$ ) and appendage areas $(6.7(2.2) v 4.8(4.9) \mathrm{cm}$; $95 \%$ CI 0.4 to $3.5 ; P=0.02)$, lower left atrial appendage emptying fractions (33 (11)\% v 52 (11)\%; 95\% CI 8 to 29; $P=$ 0.008 , and also lower left atrial appendage emptying velocities $(0.44$ $(0.21)$ v $0.79(0.27) \mathrm{m} / \mathrm{s} ; 95 \%$ CI 0.13 to $0.56 ; P=0.005)$. In addition, a higher incidence of spontaneous echo contrast $(11 \% v 36 \%)$ was observed in patients with atrial flutter and intermittent atrial fibrillation.

Conclusions-Left atrial appendage function is depressed and spontaneous echo contrast more frequent in patients with atrial flutter and intermittent atrial fibrillation, as opposed to patients with pure atrial flutter. These data support the concept that patients with atrial flutter and intermittent atrial fibrillation have an increased risk of thromboembolic events and should therefore receive adequate anticoagulant treatment.

(Heart 1997;78:250-254)
Keywords: atrial flutter; left atrial appendage; thromboembolism

The risk of thromboembolic events is assumed to be lower in patients with atrial flutter than in patients with atrial fibrillation. ${ }^{1}$ The reason for a lower risk in these patients has been attributed to the presence of synchronous atrial mechanical contraction and organised left atrial appendage function. This supposedly prevents stasis of the blood in the atrial chamber and appendage, thereby reducing the likelihood of the development of thrombi. ${ }^{2}$ Accordingly, patients with both atrial flutter and additional intermittent atrial fibrillation are considered to have a higher risk of embolic events than patients with pure atrial flutter.

Recently it has been shown that transoesophageal echocardiography may be used to assess left atrial appendage function and blood flow in the left atrium. ${ }^{3}$ In patients with atrial fibrillation, reduced left atrial appendage velocities, and the presence of spontaneous echo contrast, an echogenic swirling pattern of blood flow-indicating a low flow state in the left atrium - have been shown to be markers of thromboembolic risk. ${ }^{45}$ However, in contrast to patients with atrial fibrillation, there have as yet been no systematic studies assessing left atrial appendage function and spontaneous echo contrast in patients with atrial flutter.

The aim of this study was to describe left atrial appendage function in patients with atrial flutter and to determine whether left atrial appendage function and the incidence of spontaneous echo contrast differ between patients with pure atrial flutter and patients with atrial flutter and intermittent atrial fibrillation.

\section{Methods}

\section{STUDY PATIENTS}

The study group consisted of 20 consecutive non-anticoagulated adult patients with atrial flutter. All patients were followed up for 12 months in our outpatient clinic to determine the presence of intermittent atrial fibrillation. Holter monitoring was performed on a three months schedule and upon the occurrence of symptoms. Eleven patients in sinus rhythm without cardiac disease served as a control group (eight female, three male, age 63 (SD 3) years). After the follow up period both transthoracic and transoesophageal echocardiography were performed after written informed 
Patient characteristics and clinical data

\begin{tabular}{lll}
\hline & Atrial flutter & $\begin{array}{l}\text { Atrial flutter with } \\
\text { intermittent } \\
\text { atrial fibrillation }\end{array}$ \\
\hline Number & 9 & 11 \\
Men/women & $7 / 2$ & $8 / 3$ \\
Age (years) & $66(5)$ & $67(9)$ \\
Duration of last episode (days) & $6(6)$ & $4(4)$ \\
Heart rate (beats/min) & $89(40)$ & $98(36)$ \\
Aetiology & & 2 \\
$\quad$ Valvar disease & & 2 \\
$\quad$ Coronary heart disease & 8 & 3 \\
$\quad$ Cardiomyopathy & 1 & $2^{\star}$ \\
$\quad$ Idiopathic & & 1 \\
\hline
\end{tabular}

${ }^{\star} \mathrm{P}<0.05$ patients with pure atrial flutter $v$ patients with atrial flutter and intermittent atrial fibrillation. consent had been obtained from all patients during an episode of atrial flutter.

\section{ECHOCARDIOGRAPHIC STUDIES}

All studies were conducted with commercially available equipment (Vingmed $800 \mathrm{C}$, Vingmed Sound, Horton, Norway). To allow off-line quantitative assessment of the echocardiographic data, studies were recorded on videotape with selected cineloops and velocity spectra digitally transferred to a Macintosh Power PC computer for subsequent analysis, as described previously. ${ }^{6}$

For transthoracic echocardiography, a 3.25 $\mathrm{MHz}$ transducer was used and all patients were examined in the left lateral decubitus position. A one-lead electrocardiogram was recorded continuously. The $M$ mode left atrial dimension was measured at end systole in the parasternal long axis view, and left ventricular ejection fraction was determined according to the recommendations of the North American Society of Echocardiography. ${ }^{7}$

Topical lignocaine spray and viscous lignocaine solution were used to anaesthetise the oropharynx before transoesophageal echocardiography, which was performed with a 5 MHZ multiplane transducer. The imaging plane and gain settings were adjusted to achieve optimal visualisation of the appendage and of spontaneous echo contrast. The sample volume of the pulsed Doppler was placed $1 \mathrm{~cm}$ into the orifice of the left atrial appendage and the profile of the velocities recorded. Care was taken to minimise the angle of the incidence of the Doppler beam for flow assessment. Cineloops of the left atrium and the left atrial appendage were stored.

\section{ECHOCARDIOGRAPHIC DATA ANALYSIS}

Echocardiographic evaluations were performed in a single blinded manner, with the results confirmed by two independent observers following the original examination. The data were analysed by means of the evaluation software provided by the manufacturer (Echodisp, Vingmed Sound, Horton, Norway). The cineloops of the left atrium and left atrial appendage were examined for thrombi and the presence of spontaneous echo contrast. Left atrial appendage area was measured before cardioversion by tracing a line starting from the top of the limbus of the left upper pulmonary vein along the appendage's endocardial border. Maximum appendage area was determined during five heart cycles and averaged. The pattern of the left atrial appendage velocity profile was described and peak emptying and filling wavelets were measured during seven consecutive cycles each, and maximum velocities then averaged. In sinus rhythm, five peak velocities were averaged. Since peak emptying and filling velocities did not differ significantly, only emptying velocities are presented. Left atrial appendage ejection fraction was calculated using the following formula: $\left(\mathrm{LAA}_{\max }-\mathrm{LAA}_{\min }\right) / \mathrm{LAA}_{\max }$, where $\mathrm{LAA}_{\max }$ and $\mathrm{LAA}_{\text {min }}$ are the maximum and minimum left atrial appendage area.

\section{STATISTICAL ANALYSIS}

Descriptive data are presented as the mean (SD). Continuous variables between groups were compared by Mann-Whitney $U$ test for unpaired observations. Nominal data were compared by the Fisher's exact test. A P value $\leqslant 0.05$ was considered statistically significant (StatView 4.0, Abacus Inc, Berkeley, California, USA).

\section{Results}

PATIENTS

In 11 patients, intermittent atrial fibrillation was documented by Holter monitoring (mean
Figure 1 Left atrial appendage flow velocity profile in sinus rhythm $(A)$ and in a patient with atrial flutter (B).

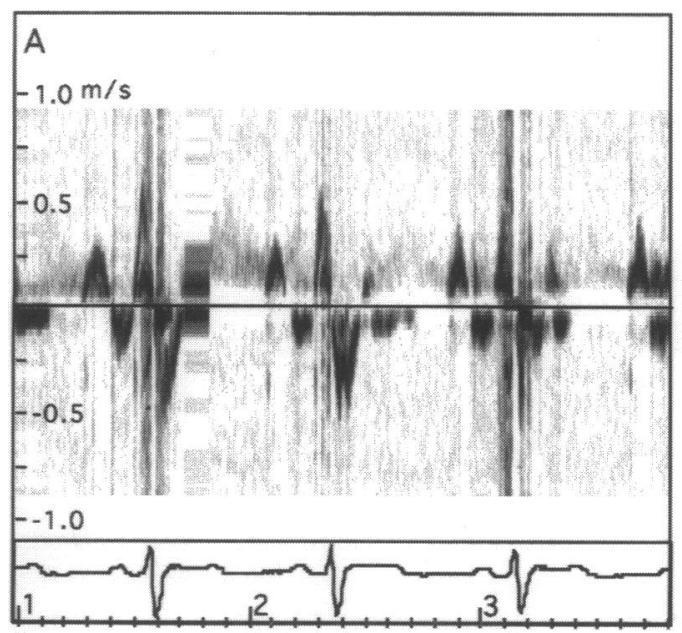



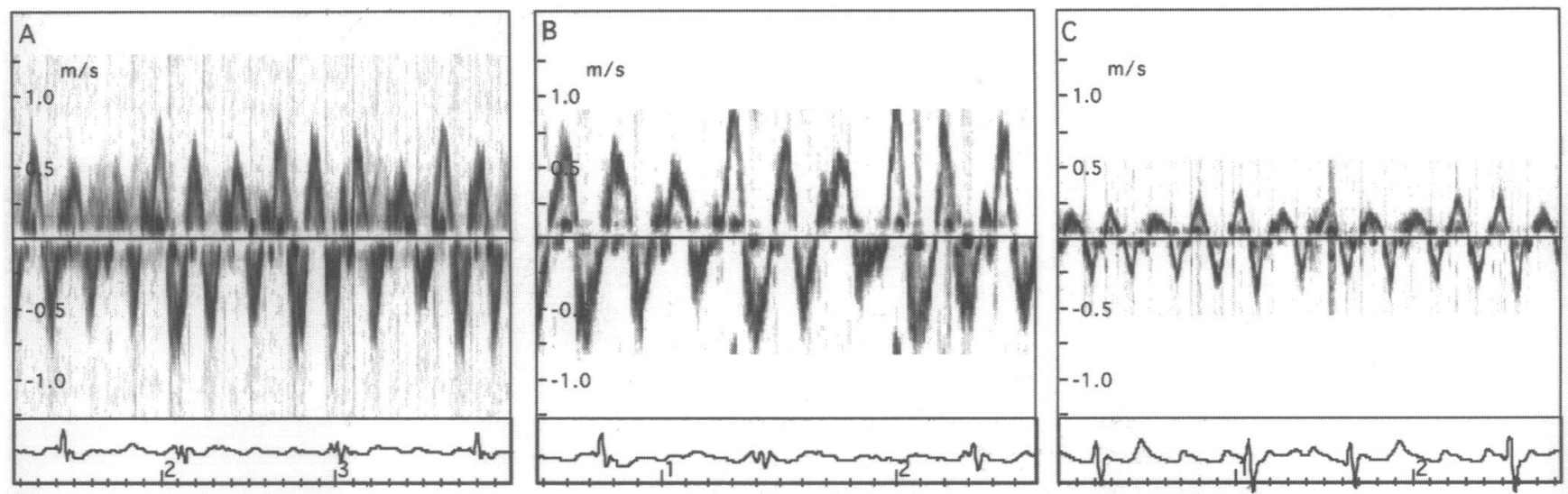

Figure 2 Left atrial appendage flow velocity profile in a patient with pure atrial flutter and an atrioventricular conduction rate of 2:1 (A), in a patient with varying atrioventricular conduction rate (B), and in a patient with atrial flutter and intermittent atrial fibrillation (C).

(SD) $1.5(0 \cdot 7)$ episodes). The other nine patients did not show atrial fibrillation and Holter monitoring was negative during the follow up period. Five patients of the latter group underwent successful catheter ablation of atrial flutter. None of these patients had palpitations during a follow up period of six months. Data of both groups are provided in the table.

LEFT ATRIAL CHAMBER AND APPENDAGE SIZE, LEFT VENTRICULAR EJECTION FRACTION, AND INCIDENCE OF SPONTANEOUS ECHO CONTRAST Patients with pure atrial flutter and controls did not differ for left ventricular ejection fraction (64 (5) \% v $62(9) \%$, NS), left atrial diameter $(3.8(0.5)$ v $3.6(0.4) \mathrm{cm}, \mathrm{NS})$, and left atrial appendage area $\left(4 \cdot 8(4.9) v 5 \cdot 0(5 \cdot 0) \mathrm{cm}^{2}\right.$, NS).

In contrast, compared with patients with pure atrial flutter, patients with atrial flutter and intermittent atrial fibrillation had significantly larger left atria $(4.5(0.6)$ v $3.8(0.5) \mathrm{cm} ; 95 \%$ confidence interval (CI) 0.2 to $1.2 ; \mathrm{P}=0.01$ ) and left atrial appendages $(6.7(2 \cdot 2)$ v $4.8(4.9)$ $\mathrm{cm} ; 95 \%$ CI 0.4 to $3.5 ; \mathrm{P}=0.02)$, and lower left ventricular ejection (45 (5)\% v 64 (5) \%, $95 \%$ CI 13 to $23 ; \mathrm{P}=0.003$ ).

Spontaneous echo contrast was not observed in the left atrial chamber and appendage in the control group. One patient with pure atrial flutter had spontaneous echo contrast in the left atrium. On the other hand, four of 11 patients with atrial flutter and intermittent atrial fibrillation had spontaneous echo contrast in the left atrium and appendage. Thrombi were not found in patients with atrial flutter or in the control group.

QUALITATIVE DESCRIPTION OF THE LEFT ATRIAL APPENDAGE VELOCITY PROFILE

In control subjects the profile of left atrial appendage velocities showed a quadriphasic pattern with two emptying and two filling waves (fig 1A). The larger of emptying waves occurred after the $\mathrm{P}$ wave on the electrocardiogram.

Patients with atrial flutter showed a different profile of left atrial appendage velocities. The pattern consisted of alternating emptying and filling wavelets (fig 1B). The number of emptying and filling wavelets during one heart cycle coincided with the number of flutter waves in the same heart cycle and was determined by the atrioventricular conduction rate. In four patients with an atrioventricular conduction rate of $4: 1$ there were four emptying and filling waves during one heart cycle, whereas in 13 patients with a $3: 1$ or $2: 1$ atrioventricular conduction rate there were three or two filling and emptying waves, respectively. In three patients with irregular atrioventricular conduction rate, the number of left atrial appendage emptying and filling waves varied in congruence to the atrioventricular conduction rate (fig 2).
Figure 3 Left atrial appendage emptying fraction $(A)$ and left atrial appendage velocities (B) in patients with simus rhythm (SR), those with pure atrial flutter (AFlu), and those with atrial flutter and intermittent atrial fibrillation $(A F l u+A F)$. LAAEF (AFlu $+A F)$. $L A A$
left atrial appendage emptying fraction; $L A A v$, left atrial appendage emptying velocities.
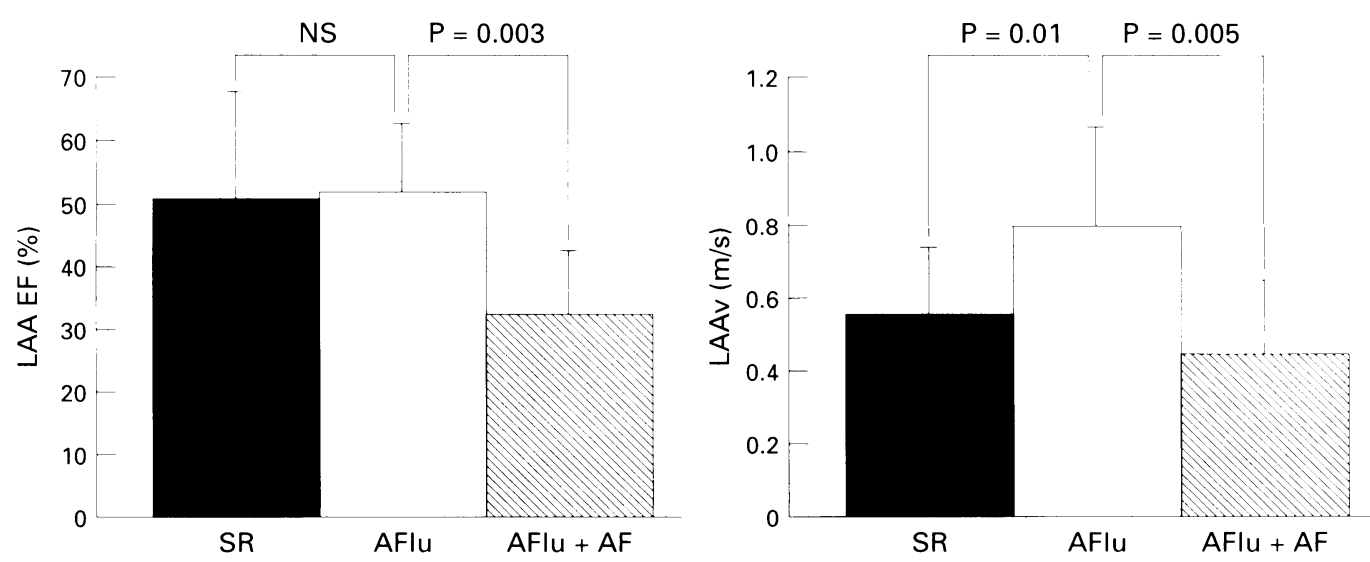
QUANTITATIVE DESCRIPTION OF LEFT ATRIAL APPENDAGE CONTRACTION AND THE LEFT ATRIAL APPENDAGE VELOCITY PROFILE

Peak emptying velocities of the left atrial appendage were significantly higher in patients with pure atrial flutter than in control subjects $(P=0.01)$ or in patients with atrial flutter and intermittent atrial fibrillation $(P=0.005)$ (fig 3). Left atrial appendage emptying fraction did not differ between patients with pure atrial flutter and control subjects. However, left atrial appendage emptying fraction was significantly lower in patients with atrial flutter and intermittent atrial fibrillation than in patients with pure atrial flutter $(P=0.008)$ (fig 3).

\section{Discussion}

The risk of thromboembolic events is assumed to be lower in patients with atrial flutter than in patients with atrial fibrillation. ${ }^{1}$ It is speculated that the lower risk of embolic events in patients with atrial flutter is due to organised mechanical atrial chamber and appendage function which prevents the formation of thrombi. However, to the best of our knowledge there have not yet been systematic studies analysing left atrial appendage function in patients with atrial flutter and comparing patients with pure atrial flutter to patients with intermittent atrial fibrillation.

\section{LEFT ATRIAL APPENDAGE FUNCTION IN ATRIAL} FLUTTER

Recently it has been shown that left atrial appendage function may be assessed by transoesophageal echocardiography. Atrial fibrillation results in a fibrillatory pattern of the left atrial appendage flow velocity profile and may result in depressed left atrial appendage contraction. ${ }^{3}$ In contrast, atrial flutter is associated with a regular pattern of atrial contraction resulting in an organised saw-type pattern of the left atrial appendage flow with alternating filling and emptying wavelets of the left atrial appendage. ${ }^{8}$ The results of our study endorse these previous observations. In both the study of Santiago et $a l^{8}$ and our study the flow wavelets coincide with the flutter waves on the surface electrocardiogram and vary with the atrioventricular conduction rate, indicating that emptying and filling flows of the left atrial appendage are caused by active atrial chamber and appendage contraction. This assumption is supported by the finding that Fourier transformation of the pulsed Doppler tracings of the left atrial appendage in atrial flutter shows a close correlation between the dominant peak frequency and the flutter emptying wave cycle length. ${ }^{9}$ However, it is likely that passive filling and emptying of the left atrial appendage may also occur, since peak flow velocities varied and decreased slightly at the end of diastole. Thus the results of this study confirm the assumption that atrial flutter causes an organised contraction of the left atrial chamber and appendage. Furthermore, the results of this study provide evidence that left atrial appendage function is similar between patients with pure atrial flutter and healthy subjects in sinus rhythm, since left atrial appendage emptying fractions did not differ between both groups. In addition, we were able to show that peak emptying velocities of the left atrial appendage are even higher in patients with pure atrial flutter than in patients with sinus rhythm.

COMPARISON OF PATIENTS WITH PURE ATRIAL FLUTTER AND PATIENTS WITH ATRIAL FLUTTER AND INTERMITTENT ATRIAL FIBRILLATION

The results of this study show that patients with pure atrial flutter and patients with atrial flutter and intermittent atrial fibrillation both had an organised pattern of left atrial appendage flow. However, in contrast to patients with pure atrial flutter and healthy subjects in sinus rhythm, peak emptying velocities and emptying fractions of the left atrial appendage were lower in patients with atrial flutter and intermittent atrial fibrillation. These findings suggest that left atrial appendage function is depressed in the latter patient group. Our results also show that patients with atrial flutter and intermittent atrial fibrillation have larger left atria and left atrial appendages. It may be assumed that the increase in size of the left atrium and left atrial appendage is caused by depressed left atrial chamber and appendage function. However, it is also possible that enlargement itself may cause depressed left atrial appendage function. The assumption is supported by the fact that most patients with atrial flutter and intermittent atrial fibrillation suffer from underlying heart disease and show decreased left ventricular ejection fractions. Furthermore, we were able to demonstrate a higher incidence of spontaneous echo contrast in patients with atrial flutter and intermittent atrial fibrillation. Recently it has been shown that spontaneous echo contrast is associated with blood stasis and thrombus formation in the left atrial chamber and appendage. ${ }^{1011}$ Therefore, spontaneous echo contrast has been suggested as a marker of increased thromboembolic risk.

No thrombus was found in our study group. However, $36 \%$ of patients with atrial flutter and intermittent atrial had spontaneous echo contrast in the left atrium. It may be assumed that the pre-existing thromboembolic milieu in the left atrial chamber and appendage may be aggravated during episodes of atrial fibrillation. Alternatively, it may also be possible that thrombi form during atrial flutter as a consequence of the thromboembolic milieu. A recent report of the finding of a thrombus in one of seven patients with atrial flutter does not mention whether any of the patients had intermittent atrial fibrillation. ${ }^{12}$

\section{CLINICAL IMPLICATIONS}

In contrast to patients with pure atrial flutter, patients with atrial flutter and intermittent atrial fibrillation are prone to depressed left atrial appendage function and often show spontaneous echo contrast in the left atrium Thus the findings of this study support the concept that patients with intermittent atrial 
fibrillation have a higher risk of thromboembolic events and should receive adequate anticoagulant treatment.

Because of the risk of thromboembolic complications in patients with atrial flutter and intermittent atrial fibrillation, the current American College of Chest Physicians guidelines state that anticoagulation should be given to this particular patient group before cardioversion. ${ }^{13}$ However, occasionally it may be difficult to decide if intermittent atrial fibrillation is present. Our study suggests that transoesophageal echocardiography may be performed in this setting to determine left atrial appendage function and to search for spontaneous echo contrast, in order to assess the risk of thromboembolism before cardioversion.

\section{STUDY LIMITATIONS}

The number of patients investigated was limited; however, measurements were assessed on a prospective basis. Although patients were prospectively followed up by Holter monitoring for determining intermittent atrial fibrillation, short or asymptomatic episodes of atrial fibrillation may have been missed. Assessing the presence of spontaneous echo contrast is subjective; however, it has been shown recently that interobserver variability in the diagnosis of spontaneous echo contrast is low. ${ }^{14}$

1 Arnold AZ, Mick MJ, Mazurek RP, Loop FD, Trohman RG. Role for prophylactic anticoagulation for direct current cardioversion in patients with atrial fibrillation or atrial flutter. $\mathcal{F} \mathrm{Am}$ Coll Cardiol 1992;19:851-5.
2 Kinch JW, Davidoff R. Prevention of embolic events after cardioversion of atrial fibrillation. Arch Intern Med 1995;155:1353-60.

3 Pollick C, Taylor D. Assessment of left atrial appendage function by transesophageal echocardiography. Implicafunction by transesophageal echocardiography. Implica-
tions for the development of thrombus. Circulation 1991; 84:223-31.
tions for the

4 Leung DYC, Black IW, Cranney GB, Hopkins AP, Walsh WF. Prognostic implications of left atrial spontaneous echo contrast in nonvalvu

5 Jones EF, Calafiore P, McNeil JJ, Tonkin AM, Donnan GA. Atrial fibrillation with left atrial spontaneous contrast detected by transesophageal echocardiography is a potent risk factor for stroke. Am $\mathcal{f}$ Cardiol 1996;78: 425-9.

6 Omran H, Jung W, Rabahieh R, Schimpf R, Wolpert C, Hagendorff A, et al. Left atrial chamber and appendage function after internal atrial defibrillation: a prospective and serial transesophageal echocardiographic study. $\mathcal{F} \mathrm{Am}$ Coll Cardiol 1997;29:131-8.

7 Henry WL. Report of the American Society of Echocardiography Committee on nomenclature and standards in two-dimensional echocardiography. Circulation 1980; 62:212-17.

8 Santiago D, Warshofsky M, Mandri GL, Tullio MD, Coromilas J, Reiffel J, et al. $\mathfrak{f}$ Am Coll Cardiol 1994;

9 Grimm RA, Chandra S, Klein AL, Stewart WJ, Black IW, Kidwell GA, et al. Characterization of left atrial appendage Doppler flow in atrial fibrillation and flutter by Fourier analysis. Am Heart f 1996;132:286-96.

10 Black IW, Hopkins AP, Lee LCL, Walsh WF, Jacobson BM. Left atrial spontaneous echo contrast: a clinical and echocardiographic analysis. $f \mathrm{Am}$ Coll Cardiol 1991;18: 398-404.

11 Fatkin D, Kelly RP, Feneley MP. Relations between left atrial appendage blood flow velocity, spontaneous echocardiographic contrast and thromboembolic risk in vivo. $\mathcal{F}$ Am Coll Cardiol 1994;23:961-9.

12 Black IW, Hopkins AP, Lee LCL, Walsh WF. Evaluation of transesophageal echocardiography before cardioversion of atrial fibrillation and flutter in nonanticoagulated patients. Am Heart f 1993;126:375-81.

13 Laupacis A, Albers G, Dunn M, Feinberg W. Antithrombotic therapy in atrial fibrillation. Chest 1992;102 (suppl):426-33S.

14 Kronik G, Stöllberger C, Schuh M, Abzieher F, Slany J, Schneider B. Interobserver variability in the detection of spontaneous echo contrast, left atrial thrombi, and left atrial appendage thrombi by transesophageal echocardioatrial appendage thrombi by transe
graphy. Br Heart $₹$ 1995;74:80-3. 